\title{
La Comarca: un instrumento lúdico para el aprendizaje y un proyecto transversal multiescuela en la Universitat Politècnica de València (UPV)
}

Eric Gielen $^{a}$, Asenet Sosa Espinosa ${ }^{\text {, }}$ José Sergio Palencia Jiménez ${ }^{c}$, Yaiza Pérez Alonso ${ }^{\text {, }}$, María Salomé Moreno Navarro ${ }^{\mathrm{e}}$, Rafael Ramón Temes Córdovez, José Luis Miralles Garcia ${ }^{\mathrm{g}}$, David Roldán Garrote ${ }^{\mathrm{h}}$, Fernando García Granada; Ana Martí Testón ${ }^{\mathrm{j}}$; Lorena Rodríguez Mattalía ${ }^{\mathrm{k}}$; Sergio Rodríguez Valdunciel'; Alvaro Sanchis Gandia ${ }^{\mathrm{m}}$; Josefa María Zarraga Llorens ${ }^{\mathrm{n}}$.

aaDto. Urbanismo, egielen@urb.upv.es; ${ }^{\mathrm{b}}$ Dpto. Urbanismo, assoes@urb.upv.es; ${ }^{\mathrm{c}}$ Dpto. Urbanismo, ipalenci@urb.upv.es; ${ }^{\mathrm{d} D p t o .}$ Urbanismo, yaipeal@urb.upv.es; ${ }^{\mathrm{e}} \mathrm{Dpto}$. Urbanismo, smoreno@upvnet.upv.es; ${ }^{\mathrm{f}} \mathrm{Dpto}$. Urbanismo, rtemesc@urb.upv.es; ${ }^{g}$ Dpto. Urbanismo, jlmirall@urb.upv.es; ${ }^{h} D C A D H A$, darolgar@har.upv.es; ${ }^{i} D S I C$, fgarcia@dsic.upv.es; ${ }^{j} D C A D H A,{ }^{j}$ anmartes@upvnet.upv.es; ${ }^{k}$ Dpto. Escultura, lorod@esc.upv.es; ${ }^{\mathrm{l} D p t o .}$ Dibujo, serrodva@doctor.upv.es; ${ }^{\mathrm{m}}$ Dpto. Pintura, alsangan@upv.es; ${ }^{\mathrm{n}}$ Dpto. Escultura, jozarllo@esc.upv.es.

\section{Abstract}

This communication is the result of an Educational Innovation and Improvement Project (PIME), financed by the Vicerrectorado de Estudios, Calidad y Acreditación of Universitat Politècnica de València, and by the School of Civil Engineering. The objective of the PIME presented in this communication is to find a new educational resource to solve motivation and learning problems in university education related to the discipline of Urbanism and Territorial Planning.

The use of the game allows to motivate and teach, and under this premise, it is proposed the creation of a strategy game of cooperation-opposition, La Comarca, designed as a simple board representing the territory, divided into four municipalities, in which four mayors interact and work to achieve a well-organized territory.

This communication describes the construction process of the game, from its idea to its production, including the design process, which is the result of a transversal project between two schools of the Universitat Politècnica de València (Escuela Técnica Superior de Ingeniería de Caminos, Canales y Puertos and the Facultad de Bellas Artes) and the main results obtained from this experience.

The first results show that the game created constitutes a useful didactic resource, allowing to present in a simple way many of the basic concepts of the discipline, so that the students work and assimilate complex concepts successfully, in a different and motivating teacher-student context.

Keywords: innovation, learning, motivation, resource, transverse, playing, gamification, teamwork, urbanism, land planning

\section{Resumen}

Esta comunicación es el resultado de un Proyecto de Innovación y Mejora Educativa (PIME), financiado por el Vicerrectorado de Estudios, Calidad y Acreditación de la Universitat Politècnica de València, y por la Escuela Técnica Superior de Ingeniería de Caminos, Canales y Puertos. El objetivo del PIME que se presenta en esta comunicación es encontrar un nuevo recurso educativo para resolver problemas de motivación y aprendizaje en la enseñanza universitaria relacionada con la disciplina del Urbanismo y la Ordenación del Territorio. 
El uso del juego permite motivar y enseñar, y bajo esta premisa, se propone la creación de un juego de estrategia de cooperación-oposición, La Comarca, diseñado como un sencillo tablero representando el territorio, dividido en cuatro municipios, en el que cuatro alcaldías interactúan y trabajan para conseguir un territorio bien organizado.

En esta comunicación se describe el proceso de construcción del juego, desde su idea hasta su producción, pasando por el proceso de diseño, fruto de un proyecto transversal entre dos escuelas de la Universitat Politècnica de València (Escuela Técnica Superior de Ingeniería de Caminos, Canales y Puertos y la Facultad de Bellas Artes) y los principales resultados obtenidos tras dicha experiencia.

Los primeros resultados muestran que el juego creado constituye un recurso didáctico útil, permitiendo presentar de manera sencilla muchos de los conceptos básicos de la disciplina, para que el alumnado trabaje y asimile conceptos complejos con éxito, en un contexto profesoradoalumnado diferente y motivador.

Palabras clave: innovación, aprendizaje, motivación, recurso, transversal, juego, gamificación, trabajo en equipo, urbanismo, ordenación del territorio

\section{Introducción}

La gamificación en la educación se centra en el uso de juegos para motivar a los estudiantes a involucrarse activamente con el tema central de la actividad gamificada (Paisley, 2013).

Siendo pues la motivación un elemento esencial en el proceso de enseñanza-aprendizaje, el recurso lúdico constituye una herramienta excelente para construir una relación distinta entre profesor y alumnado y reforzar la motivación por aprender, tal y como dicen Rodríguez, F., \& Santiago, R. (2015).

Existen numerosas experiencias documentales que muestran como la gamificación puede constituir una herramienta útil que permita enseñar, experimentar y disfrutar de una forma entretenida y práctica (Alcaide y De la Poza, 2019; Serna et al., 2016; González, 2014; Roig-Vila, 2019). El aprendizaje mediante el juego es proactivo, atractivo, enriquece los conocimientos al tiempo que la participación y la motivación aumentan (Chacon, 2008; Serna et al., 2016). Sin embargo, las experiencias sobre gamificación, en la literatura más reciente consultada, parecen estar más ligadas al uso de las nuevas tecnologías (Cano-Terriza et al. 2019; Castañeda-Vázquez et al. 2019; Ríos et al. 2019) o de juego tipo escape room (Pérez Vázquez, 2019), que juegos más tradicionales como el que se propone en esta comunicación.

El juego es un sistema formal basado en reglas con un resultado variable y cuantificable, donde, por un lado, diferentes resultados se asignan a distintos valores y, por otro, el jugador debe hacer un esfuerzo para influir en el resultado. El efecto para el jugador es que éste se siente unido al resultado, ya que las consecuencias de la actividad son opcionales y negociables (Juul, 2003).

La utilización del juego es relativamente común en la enseñanza de las matemáticas en edades tempranas, sin embargo, su presencia es muy limitada en el ámbito universitario (Gaete-Quezada, 2011). Esto se debe a dos motivos principales: en primer lugar, al desconocimiento de los docentes en cuanto a sus ventajas como recurso de aprendizaje (Chacón, 2008); en segundo lugar, la docencia universitaria muy a menudo, se encuentra anclada en paradigmas tradicionales, donde el docente tiene un rol central y el alumno y la alumna un rol pasivo (Gaete-Quezada, 2011). El juego permite, además, romper las barreras, mejorar la comunicación en el grupo, al generar nuevas ideas, visiones y estrategias” (González, 2014). 
Con el juego se aprende a hacer, lo cual permite al alumnado la adquisición de competencias para su capacitación profesional, enfrentarse a situaciones diversas y trabajar en equipo, todo ello dentro de los marcos sociales y laborales donde desarrolla su vida (Delors, 1996).

Algunas materias, como es el caso de la Ordenación del Territorio, son conceptualmente más complicadas que otras, requieren un nivel de abstracción superior, por lo que resulta necesario buscar alternativas al aprendizaje tradicional de pizarra y clases magistrales. En este sentido, el juego constituye una gran oportunidad para la mejora del aprendizaje y particularmente en lo que se refiere a la adquisición de competencias en este tipo de disciplinas. Se trata pues de implantar una estrategia lúdica, de poner en situación al alumnado, construyendo un entorno que estimule al estudiante a crear su propio conocimiento y experiencia (Chacón, 2008). En este sentido, permite trabajar todas las fases del proyecto: establecimiento de objetivos, diagnóstico, pasando por la divergencia o generación de ideas, hasta la convergencia en soluciones prácticas. El juego en aula proporciona una herramienta que facilita la comprensión de los contenidos teóricos, pues la complejidad para comprender la teoría está vinculada con los distintos niveles de abstracción: lo intangible es más complejo de comprender que lo tangible. Así, el recurso lúdico-didáctico que aquí se propone, pretende precisamente investigar cómo llevar la complejidad territorial al aula de forma tangible (Sosa y Peréz, 2014).

La relación entre juego y aprendizaje es natural ya que desde la infancia el juego es parte del aprendizaje. Lo que ha cambiado en la actualidad es el tipo de juego. En las etapas más tempranas, el juego es material, en un soporte físico, fomentando las habilidades manuales de los niños, pero muy rápidamente con el acceso a las nuevas tecnologías se abandona este formato y se opta por juegos virtuales. Las nuevas generaciones están acostumbradas mayoritariamente a los juegos electrónicos, medios digitales y a las redes sociales, llegando a presentar cierta dependencia (Alcaide y De la Poza, 2019). Aun así, el juego de mesa resiste e incluso en la última década ha habido un boom de los juegos de tablero. Desde la editorial DEVIR afirman que "cada año se incrementan un $40 \%$ las ventas con respecto al año anterior" (Piñeiro, 2016). El resurgir de los juegos de mesa parece ser una necesidad biológica de disfrutar del ocio lejos de una pantalla, diferenciándose de una realidad laboral muchas veces inmersa en un trabajo alienante lejos de cualquier resultado físico o tangible (Piñeiro, 2016).

\section{Objetivos}

En la universidad se está produciendo un descenso en el número de matriculaciones en las carreras de ingeniería que se encuentran directamente relacionadas con la obra civil y la construcción en general. Este hecho ha supuesto un descenso en la exigencia de acceso a este tipo de titulaciones, tradicionalmente elegidas de forma mayoritaria por alumnado con un alto expediente académico. De este modo, se ha producido una modificación en el perfil del estudiante de nuevo ingreso, que ahora acceden a estas titulaciones con notas de corte inferiores, lo que, salvo excepciones, indica que el alumnado medio actual que se matricula en estas titulaciones accede con un nivel académico medio-bajo. A esto hay que sumar el hecho de que su primera opción no es siempre el título en el que se matricula, por lo que accede con una menor motivación.

Por otro lado, las asignaturas del área de conocimiento Ordenación del Territorio y Urbanismo son complejas. Se trata de una disciplina con un enfoque transversal, global e integrador, lo que supone la incorporación necesaria de un pensamiento complejo y abstracto, lo que dificulta el aprendizaje profundo de estas materias en el alumnado. La problemática se acentúa aún más en los primeros cursos, especialmente en primero y segundo curso de grado, donde el alumnado no dispone del bagaje y la 
madurez suficiente para abordar con éxito estas temáticas. Todo ello supone una dificultad añadida al aprendizaje del estudiante, a lo que hay que añadir la escasa motivación, que se pone de manifiesto en las asignaturas troncales de los primeros cursos, y que repercute a su vez en un bajo índice de matriculación en las asignaturas optativas de los cursos siguientes.

Como consecuencia del análisis anterior, nace esta propuesta de Proyecto de Innovación y Mejora Educativa (PIME). El objetivo principal es el de crear un juego de mesa como recurso lúdico-educativo para mejorar el proceso de aprendizaje en las asignaturas de Urbanismo y Ordenación del Territorio. La idea es crear un juego bajo los principios de la cooperación y la competición, en el que se ponga de manifiesto la aparición de conflictos y la necesidad de crear consenso para gestionarlos, dos características inherentes a cualquier tipo de decisiones territoriales. Se pretende así diseñar un juego de mesa para incorporarlo, en futuros cursos, en las asignaturas de la unidad docente, así como en actividades paralelas y transversales de la Escuela. En este sentido, el nuevo recurso didáctico debía ser aplicado y creativo, partiendo de la premisa siguiente: el juego proporciona una oportunidad para mejorar el proceso de enseñanza-aprendizaje dentro de disciplinas complejas y con cierto nivel de abstracción como son el urbanismo y la ordenación del territorio.

Con todo ello se plantea una serie de objetivos específicos que son:

1. Reflejar la complejidad territorial en el recurso didáctico, introduciendo los principales criterios de actuación de la ordenación del territorio, su carácter multidisciplinar y la existencia de múltiples agentes e intereses, interviniendo en un difícil compromiso de competición y colaboración por un mismo espacio, reflejando la necesidad de actuar por el bienestar común de la sociedad. Indicadores de logros: Existencia del juego de mesa, número de conceptos y criterios de ordenación del territorio reflejados en el juego.

2. Testear la idoneidad del juego como recurso didáctico para la enseñanza de la Ordenación del Territorio. Indicadores de logros y/o evidencias: Número de participantes, proporción de alumnos/as que consideran que los juegos les ayudan a entender el contenido de la asignatura y proporción de alumnos/as que afirman que a través del juego se aprende.

3. Testear la idoneidad del juego como elemento motivador del alumnado para aprender, introduciendo actividades lúdicas en el currículo del alumnado. Indicadores de logros y/o evidencias: proporción de alumnos/as que consideran que el uso del juego les motiva para el aprendizaje.

Finalmente, este proyecto busca de manera más estratégica "seducir" y atraer a los estudiantes hacia la disciplina de la Ordenación del Territorio.

\section{Desarrollo de la innovación}

El proyecto se ha desarrollado en la Escuela Técnica Superior de Ingeniería de Caminos, Canales y Puertos (ETSICCP). Los resultados del mismo van dirigidos al alumnado de las titulaciones de Grado de Ingeniería Civil (GIC), Grado en Ingeniería de Obras Públicas (GIOP), así como el Máster de Caminos, Canales y Puertos (MCCP) y Máster en Transporte, Territorio y Urbanismo (MTTU), donde el Departamento de Urbanismo tiene docencia relacionada con el área de conocimiento de Urbanismo y la Ordenación del Territorio en las asignaturas siguientes: 


\begin{tabular}{|c|c|c|c|c|}
\hline Asignatura (Código) & Titulación & Curso & $\begin{array}{r}\text { Número de estudiantes } \\
(2018-2019)\end{array}$ & $\begin{array}{r}\text { Número de estudiantes } \\
(2019-2020)\end{array}$ \\
\hline - Transporte y Territorio (12828) & GIC & $2^{\circ}$ - Troncal & 63 & 68 \\
\hline - Urbanismo y Territorio (12154) & GIOP & $2^{\circ}$ - Troncal & 71 & \\
\hline $\begin{array}{l}\text { - Ingeniería Civil para la Sociedad } \\
(13470)\end{array}$ & GIC & $4^{\circ}$ - Optativa & 27 & 38 \\
\hline $\begin{array}{l}\text { - Medio Ambiente y Desarrollo } \\
\text { Sostenible (12537) }\end{array}$ & GIOP & $4^{\circ}$ - Optativa & 5 & 2 \\
\hline - Gestión Territorial (12545) & GIOP & $4^{\circ}$ - Optativa & 5 & 1 \\
\hline $\begin{array}{l}\text { - Urbanismo y Ordenación del } \\
\text { Territorio (33545) }\end{array}$ & MCCP & $1^{\circ}$ - Troncal & 89 & 75 \\
\hline - Planificación Territorial (33519) & MCCP & $2^{\circ}$ - Optativa & 2 & 2 \\
\hline - Planificación Territorial (34591) & MTTU & $1^{\circ}$ - Troncal & 17 & 34 \\
\hline - Planeamiento Urbanístico (34593) & MTTU & $1^{\circ}$ - Troncal & 20 & 36 \\
\hline
\end{tabular}

Estas nueve asignaturas fueron incorporadas con el fin de abarcar la totalidad del currículo del área de conocimiento de Urbanismo y Ordenación del Territorio en la ETSICCP, donde existe el problema que pretende resolver este PIME.

Además, aprovechando los recursos y las posibilidades de sinergia entre escuelas de la Universitat Politècnica de València (UPV), se integró a la Facultad de Bellas Artes (BBAA) en el desarrollo metodológico del PIME, a través de un proyecto transversal realizado en el primer curso de Grado en Diseño y Tecnologías Creativas (GDTTCC). En este caso, fueron las asignaturas siguientes:

\begin{tabular}{lllr}
\hline & & & Número de estudiante \\
Asignatura (Código) & Titulación & Curso & $(2018-2019)$ \\
\hline - Fundamentos de volumen (13809) & GDTTCC & $1^{\circ}$ - Troncal & 107 \\
- Fundamentos de diseño (13811) & GDTTCC & $1^{\circ}$ - Troncal & 107 \\
- Fundamentos de la imagen digital (13810) & GDTTCC & $1^{\circ}$ - Troncal & 107 \\
\hline
\end{tabular}

Se integraron estas asignaturas del GDTTCC, ante la oportunidad que suponía el buscar el diseño más atractivo posible para el producto final y poder colaborar dentro de una universidad politécnica como es la UPV con el alumnado y el profesorado de otra Escuela con perfiles y competencias diferentes y a la vez complementarias a las de la ETSICCP.

El presente proyecto se ha desarrollado en 3 fases. A continuación, se detallan los trabajos realizados en cada una de las fases durante el periodo de 2 años que ha durado el proyecto de innovación educativa:

- FASE 1: Análisis de juegos existentes afines a la disciplina de la Ordenación del Territorio y el Urbanismo.

- FASE 2: Diseño y elaboración del juego de mesa.

- FASE 3: Testeo y validación del producto. Análisis de los resultados del aprendizaje.

\section{Fase 1}

Entre los diferentes juegos existentes en el mercado, se puede establecer las siguientes tipologías (Ripoll, 2006): Juegos individuales (en los que el jugador no tiene oponentes, juega superando hitos alcanzados en partidas anteriores); Juegos de oposición (en los que el jugador no tiene compañeros pero sí oponentes y debe jugar para superarlos); Juegos de cooperación (no los que existen oponentes, solo compañeros, y entre todos se tiene que superar un reto, buscando una estrategia común.); Juegos de cooperación- 
oposición (en los que existen compañeros y oponentes, y cada uno de los grupos juega a superar al resto buscando estrategias comunes que resulten óptimas).

En nuestro caso, el tipo de juego que nos interesaba se asimiló a un juego de cooperación-oposición, ya que las materias relacionadas con el Urbanismo y la Ordenación del Territorio poseen un enfoque multicriterio centrado en la solución de problemas, fruto de un trabajo interdisciplinario, donde intervienen múltiples agentes con intereses y propuestas de actuación diferentes, pero con un fin común, el bienestar de la sociedad.

Con enfoques similares, existen experiencias como el Modelo CLUG (Community Land Use Game), diseñado por Allan Felt (1972), FLOOD RESILIENCE GAME desarrollado por Zurich Flood Resilience Alliance y el Institute International of Applied Systems Analysis o THE GAME OF URBAN RENEWAL, creado por Variants of Evil, Flavio Trevisan.

También, en el mercado actual, se pueden encontrar numerosos juegos de mesa, orientados hacia la estrategia como HONSHU, COLONOS DE CATÁN o LA ISLA PROHIBIDA que incorporan una cierta componente territorial y multicriterio. En este mismo sentido, hay juegos video como SIMCITY o CITIES SKYLINES, generalmente muy conocidos por los jóvenes, e incluso una sencilla APP del servicio público holandés responsable del catastro MOVE A LOT.

Con el objetivo de estudiar de la relación del alumnado con juegos afines al Urbanismo y la Ordenación del Territorio y definir los criterios óptimos de diseño de nuestro propio juego, se seleccionaron y testearon cuatro juegos comerciales: HONSHÚ, LA ISLA PROHIBIDA, COLONOS DE CATÁN en su versión básica y CATÁN en su versión petróleo (Figura 1).

Para ello, se realizaron seis talleres con los profesores participantes en el proyecto y un taller con el alumnado de la Escuela de Caminos, en cual se realizaron 33 encuestas para estudiar sus hábitos y preferencias de juego.

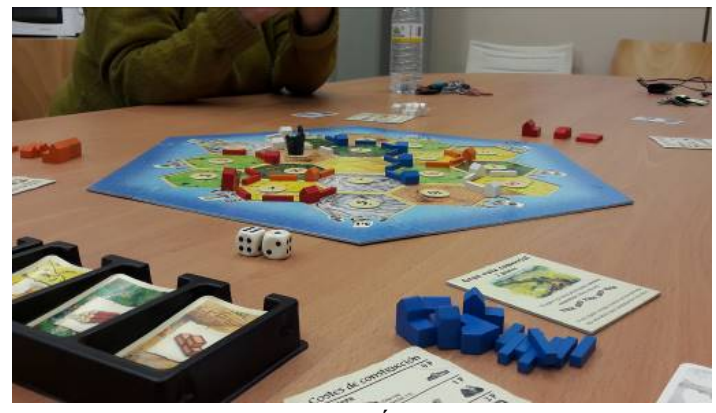

COLONOS DE CATÁN, versión básica

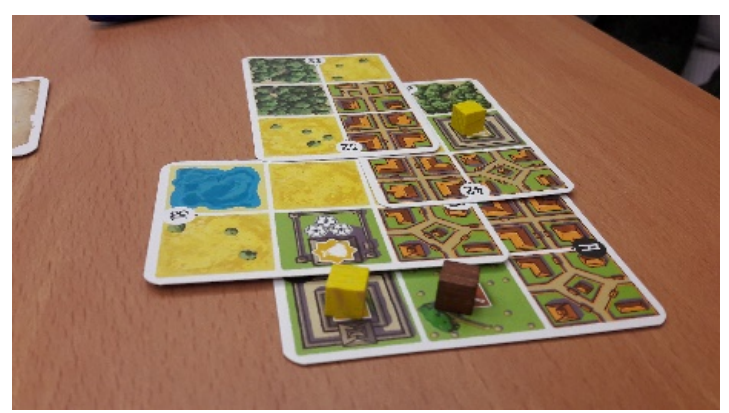

HONSHU

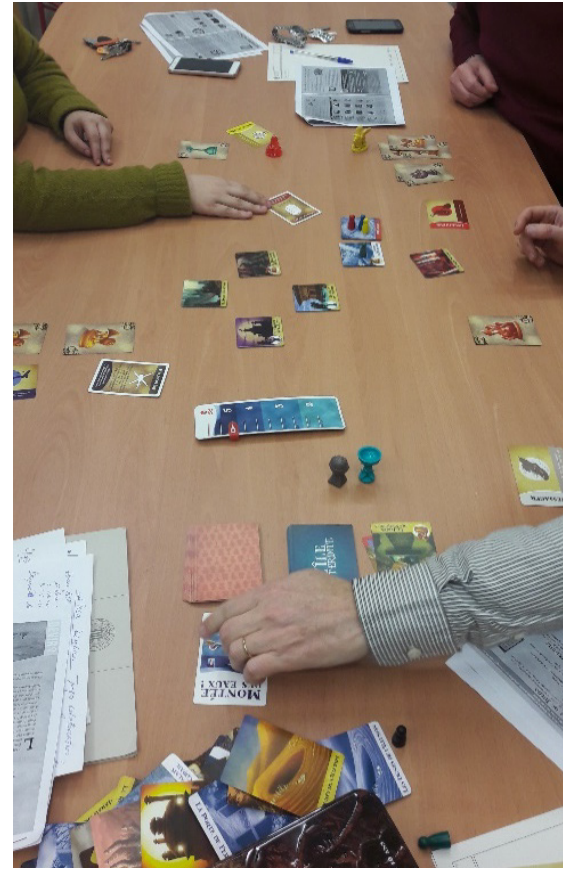

LA ISLA PROHIBIDA

Figura 1: Juegos comerciales testeados

(c)) BY-NC-ND 2020, Universitat Politècnica de València

Congreso In-Red (2020) 
La encuesta ex ante revela que al 63,6\% del alumnado le gustaría que se incorpore este tipo de recurso de aprendizaje en el ámbito universitario, mientras que al $9 \%$ no les gusta y al $27 \%$ no saben o no contestan. El 88\% de los encuestados consideran el uso del juego como un elemento motivador, puntuando con una nota superior o igual a 5, siendo 1 nada motivador y 10 totalmente motivador (Figura 2).

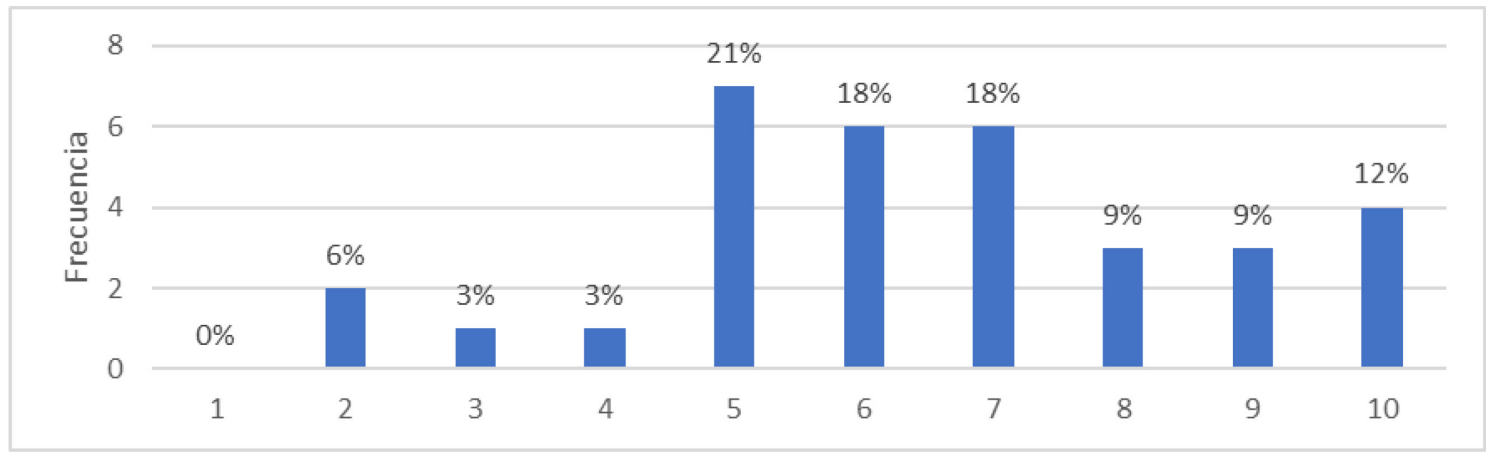

Figura 2: Valoración de 1 a 10, siendo 1 nada motivador y 10 muy motivador, el uso del juego como recurso para el aprendizaje en el ámbito universitario

De esta fase primera, se extrajeron diferentes ideas importantes. En primer lugar, el alumnado ve con interés la incorporación del juego de mesa como recurso para el aprendizaje en el ámbito universitario. En segundo lugar, se evidenció que el tipo de juego más adecuado para el fin que buscábamos era el de cooperación- oposición: era necesario introducir la competitividad entre jugadores como elemento motivador, pero estableciendo mecanismos de cooperación entre jugadores de obligado cumplimiento para ganar la partida. Como conclusión, se decidió diseñar un juego de estrategia, con un sencillo tablero representando un territorio, en el cual interactuasen distintos agentes que en todo momento intentan desarrollar sus actividades, todos ellos con distintos intereses, y a veces enfrentados. Todo ello se canaliza, mediante la asunción de los roles que representan los distintos agentes sociales que, actuando dentro de un marco definido en las propias reglas del juego, pretende reproducir los criterios básicos de lo que supone una ocupación racional y sostenible del suelo. De este modo, para ganar, los jugadores compiten entre sí para cumplir sus objetivos, pero para ello es necesario que la "sociedad" creada alcance ciertos niveles de "calidad y bienestar social", siendo necesario para ello que se trabajen valores transversales como la colaboración y la sostenibilidad.

\section{Fase 2}

Con el aprendizaje adquirido en la fase anterior y las aportaciones del profesorado en materias relacionadas con el Urbanismo y la Ordenación del Territorio, se pasó a una fase más creativa cuyo objetivo era idear una primera versión del juego de mesa. A través de múltiples reuniones de trabajo, se definieron los objetivos del juego, su lógica y los diferentes elementos que lo compondrían (Figura 3). Posteriormente, se desarrolló una versión borrador del juego, con su tablero y los distintos componentes (Figura 4), y se empezó a testear (Figura 5), arrancando así un proceso iterativo de prueba/error que permitió, poco a poco, ajustar las normas y la totalidad de los elementos que compondrían el juego. 


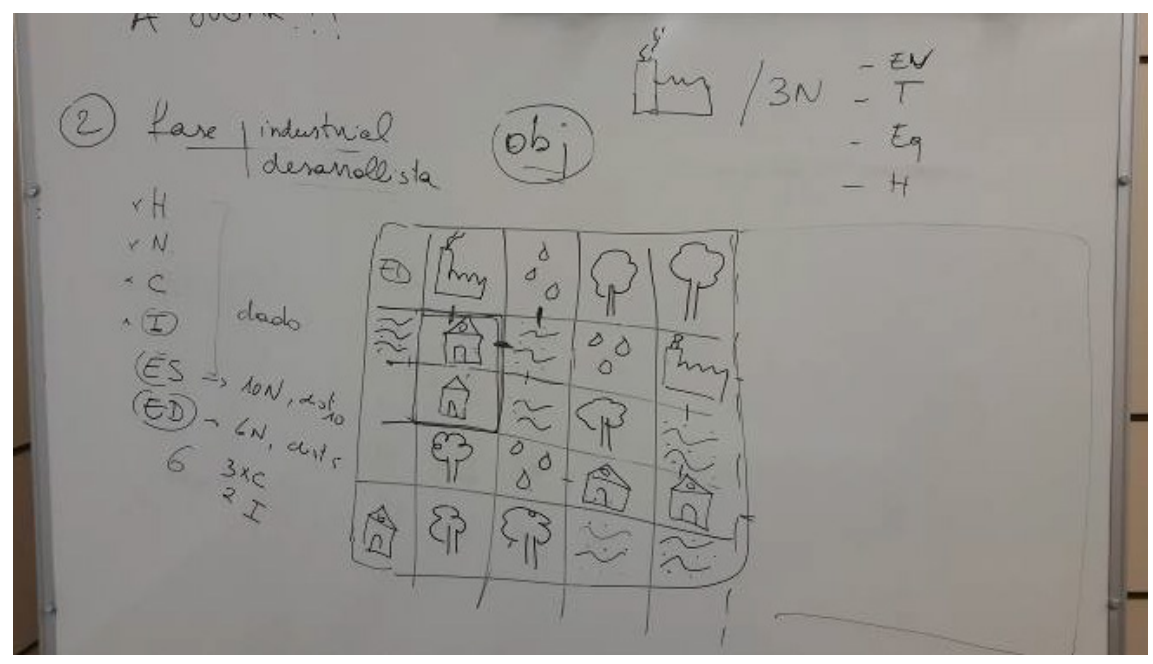

Figura 3: Proceso de elaboración del juego

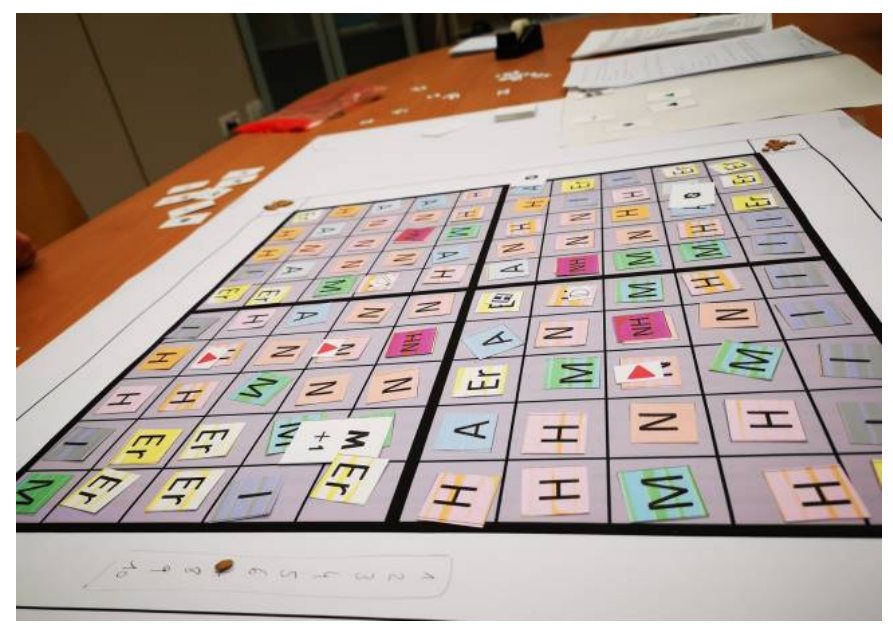

Figura 4: Primera versión borrador del juego

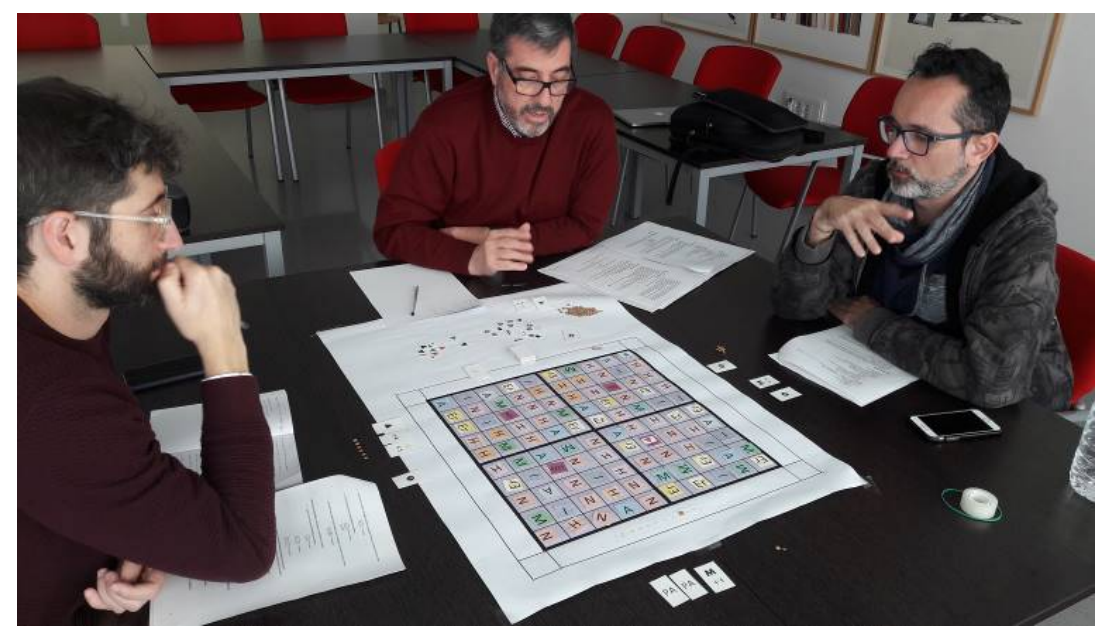

Figura 5: Testeo del juego 
Una vez testeado el juego, pasamos a la fase de diseño. En esta fase, el objetivo fue convertir la versión borrador en un producto lo más atractivo posible para el alumnado.

Aprovechando las potencialidades que ofrece un campus politécnico como el de la UPV, surgió la posibilidad de integrar la Facultad de Bellas Artes al proyecto y resolver las limitaciones que tenía el equipo docente de la Escuela de Caminos para el diseño del juego. Se presentó la propuesta a un grupo de profesores de BBAA y se decidió involucrar a los alumnos de $1^{\text {er }}$ curso de Grado en Diseño y Tecnologías Creativas (GDTTCC) a través de un proyecto transversal en el que colaborarían las asignaturas de Fundamentos de volumen, Fundamentos de diseño y Fundamentos de la imagen digital, pudiéndose de este modo completar todo el proceso de diseño y producción del juego.

El proyecto transversal se desarrolló de la siguiente manera. A principios de abril de 2019, el profesorado participante en el PIME perteneciente a la ETSICCP, actuando como cliente, presentó el "encargo" del producto a diseñar al alumnado de $1{ }^{\mathrm{er}}$ curso de GDTTCC, y entregaron el material de apoyo necesario para la realización del proyecto transversal (video con la dinámica del juego, la versión borrador, instrucciones y listado de componentes). Con la colaboración del profesorado de BBAA y, con el material entregado, se empezó a desarrollar la actividad correspondiente al proyecto transversal en las distintas asignaturas involucradas que, tras una primera parte del cuatrimestre más conceptual, dedicaron el resto de sus horas lectivas al proyecto. El proyecto transversal se organizó en 26 grupos de 4 estudiantes. En el transcurso de esta actividad, el alumnado fue aclarando dudas con el cliente (el profesorado de la ETSICCP) e incluso algunos grupos llegaron a entrevistarse con alumnado de la propia para entender mejor el perfil del jugador potencial.

El 15 de abril, los grupos de trabajo de GDTTCC entregaron un pre-proyecto. Esta entrega fue contestada unos días después con unos comentarios de retroalimentación, realizados en una reunión de coordinación/evaluación con el profesorado de BBAA y de ETSICCP (el cliente). El feedback se centró en mejorar y corregir con el alumnado tanto el diseño, la imagen de juego como su jugabilidad. También se tuvo que reorientar el diseño de algunos grupos ya que adoptaron una estética propia conforme a lo que ellos percibían que era el urbanismo, que no se correspondía con lo que el encargo requería.

Un mes después del inicio del proyecto transversal, se entregaron y presentaron públicamente al cliente 26 proyectos con maquetas incluidas (Figura 6). A continuación, vino el fallo del jurado para seleccionar el proyecto ganador. Para ello, se reunieron dos comisiones: 4 juegos fueron elegidos por el equipo de profesores ETSICCP y luego, el equipo de profesores del Grado de Diseño y Tecnologías Creativas seleccionó la propuesta con la mejor estética. 


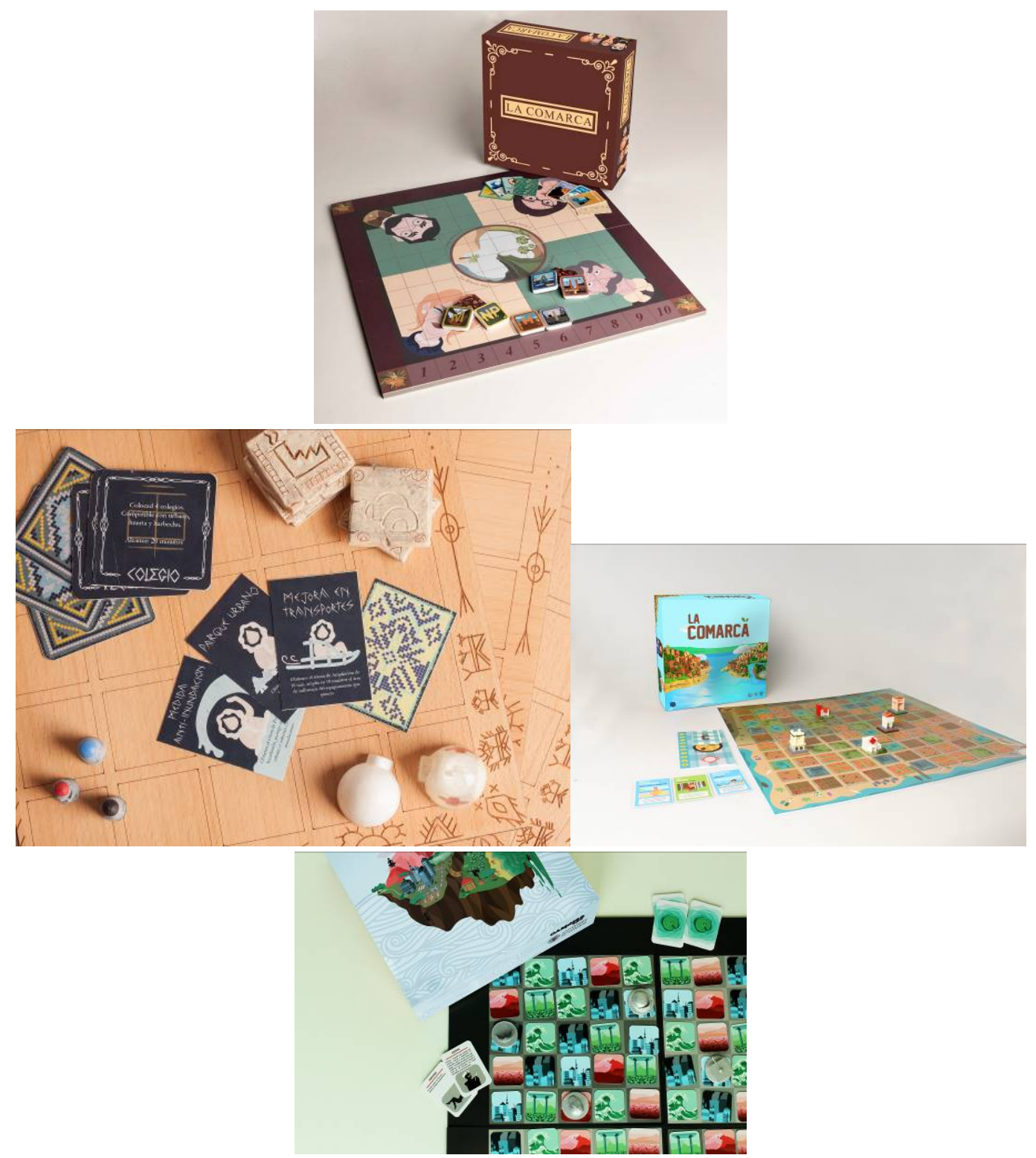

Figura 6: Ejemplos de proyectos presentados por el alumnado de BBAA

\section{Fase 3.}

Con el juego de mesa creado, el recurso lúdico se incorporó en diversas asignaturas de la ETSICCP, reservando un espacio de 2 horas de clase para llevarlo a cabo en el aula y testearlo así con el alumnado. Esta fase se vio interrumpida por el confinamiento de marzo 2020, sin embargo, aun así, se pudo realizar la actividad con 24 participantes en las asignaturas siguientes:

- Transporte y Territorio, del $2^{\circ}$ curso de Grado de Ingeniería Civil.

- Planificación Territorial, del $2^{\circ}$ curso del Máster de Ingeniería de Caminos, Canales y Puertos

- Planificación Territorial, $1^{\circ}$ curso del Máster Universitario de Transporte, Territorio y Urbanismo. 
En esta fase, para recabar aquella información que nos permita validar el juego como recurso para el aprendizaje en el aula universitaria, se han aplicado técnicas de observación directa en cada una de las mesas de juego, entrevistas semidirigida con alumnado participante en el juego y encuesta a todo el alumnado participante en las sesiones de juego. Debido al confinamiento tan solo se pudieron recoger 14 encuestas.

\section{Resultados}

El principal resultado de este proyecto es la materialización y puesta en práctica del juego de mesa $L a$ Comarca (Figura 7). Contamos con 5 tableros de 4 jugadores cada uno.

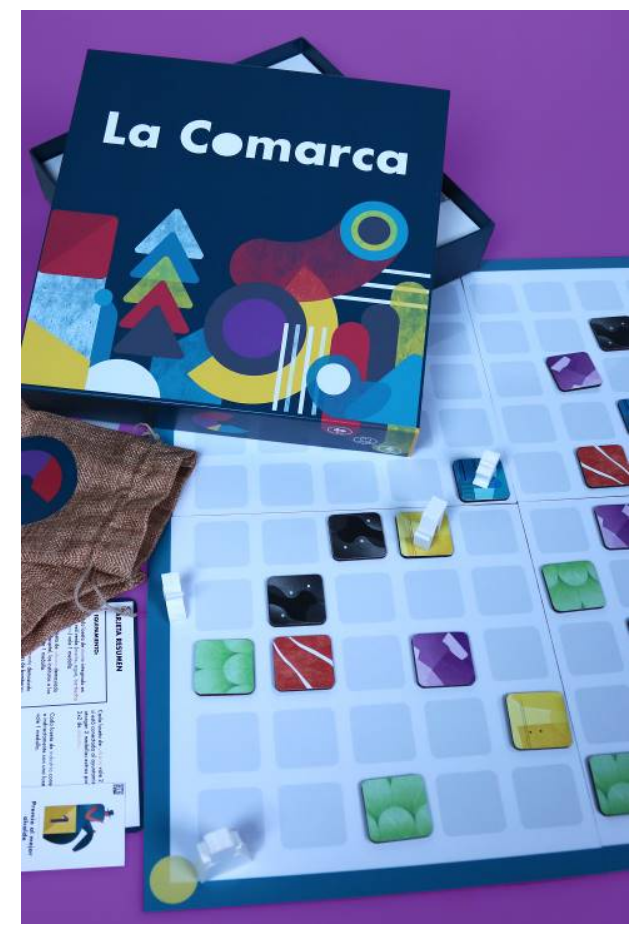

Figura 7: Maqueta del juego la Comarca

La sinopsis del juego es la siguiente:

\section{Un juego de tablero para 4 jugadores o jugadoras}

La Comarca es un juego de tablero para 4 jugadores o jugadoras. Cada uno de los 4 miembros del juego se convierte en una alcaldía que busca alcanzar el mayor bienestar de su población. Deberás administrar tu territorio lo mejor posible y colaborar con los demás en lo que se refiere a equipamientos básicos comunes.

Tu reto y el de las demás Alcaldias es conseguir el modelo territorial óptimo, pero atención, no será fácil. Hay intereses particulares y comunes que habrá que saber compaginar. Deberás hacer crecer tu ciudad y organizar los usos en el territorio de manera racional, asegurando la continuidad y compacidad del tejido urbano, procurando mantener las industrias y la huerta próxima a la ciudad y construyendo una amplia red de espacios verdes.

Y no te olvides, este crecimiento no sirve de nada si no viene acompañado de una adecuada dotación

(c)) EY-NC-ND 2020, Universitat Politècnica de València

Congreso In-Red (2020) 
en equipamientos básicos, por lo que tendrás que procurar que tu territorio esté bien atendido por los servicios públicos básicos. Tu población necesita servicios sanitarios, educativos y también proteger sus montes contra incendios, así como encontrar lugares donde verter sus residuos no valorizables o reciclables.

En cuanto al recurso lúdico creado y como consecuencia de las sesiones de juego realizadas en las diversas asignaturas de la ETSICCP (Figuras 8 y 9), se han podido analizar los resultados en relación con los objetivos específicos del proyecto. En primer lugar, se ha creado un juego eficaz que refleja la complejidad territorial, introduciendo los principales criterios de actuación de la ordenación del territorio. La estética del juego está a la altura de las opciones comerciales analizadas, por lo que consideramos que la propuesta final elegida ha sido la más adecuada para el fin que buscábamos. Segundo, se pudo confirmar la idoneidad del juego como recurso didáctico en el contexto de la enseñanza de la Ordenación del Territorio. En tercer lugar, se logró motivar al alumnado para que pusiese en práctica los conceptos estudiados en la teoría en el caso práctico del juego didáctico.

Resultó difícil simplificar la complejidad territorial y más aún, trasladarla al tablero y a las instrucciones del juego: Hubo que procurar cuidar la jugabilidad, la estética, medir correctamente el tiempo y asegurar la adquisición de aprendizajes esenciales. Hemos de reconocer que ha sido un ejercicio complicado por parte del profesorado de la ETSICCP pero al final, se ha podido crear un juego de mesa que cumple con las condiciones anteriores. Según los discursos recogidos en las sesiones del juego realizadas con el propio alumnado, se deduce que se ha conseguido reflejar parte de la complejidad territorial buscada, introduciendo de una manera intuitiva los principales criterios básicos de actuación del Urbanismo y la Ordenación del Territorio, su carácter multidisciplinar y la existencia de múltiples agentes e intereses, interviniendo y tomando decisiones en un difícil escenario que combina el compromiso, la competición y la colaboración por un mismo espacio, en busca del bienestar común de la sociedad.

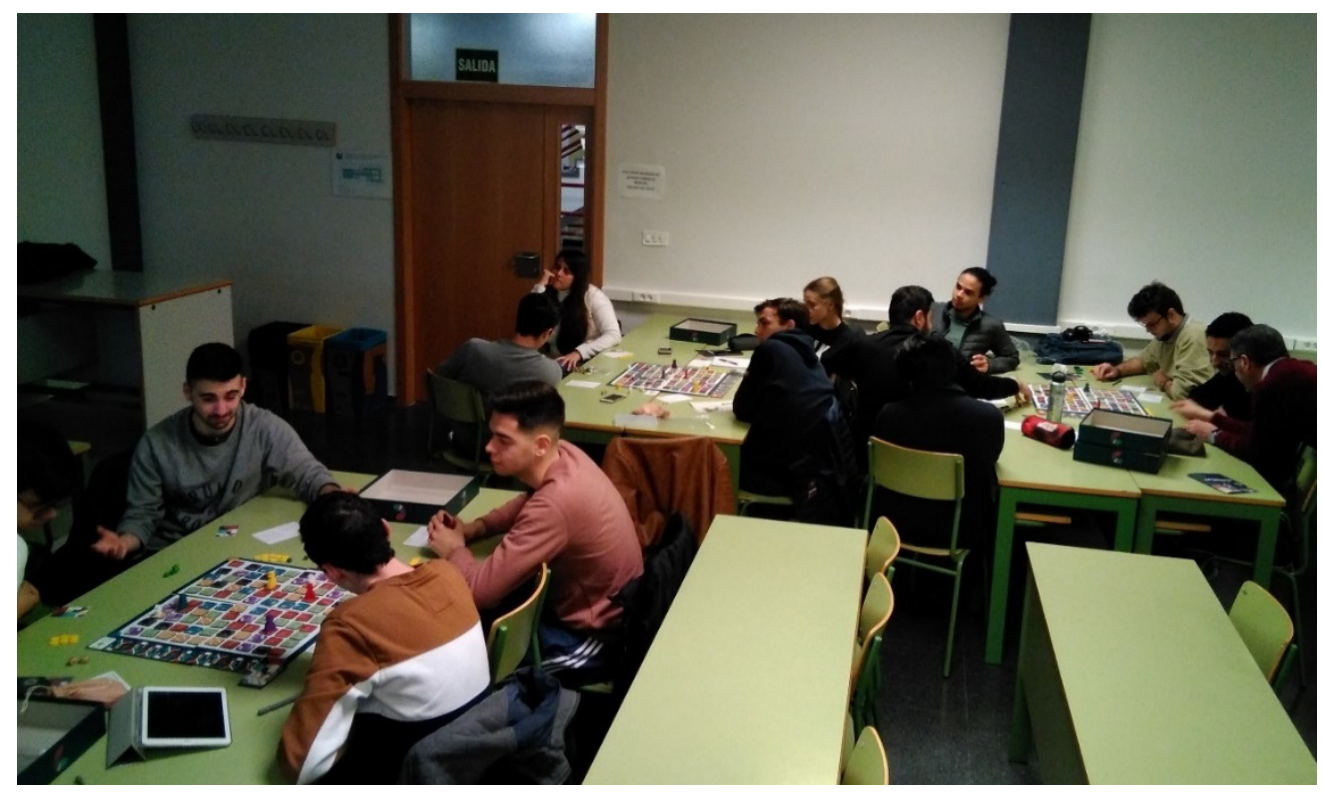

Figura 8: Sesión de juego celebrada en la asignatura Transporte y Territorio, $2^{\circ}$ curso de Grado de Ingeniería Civil. 


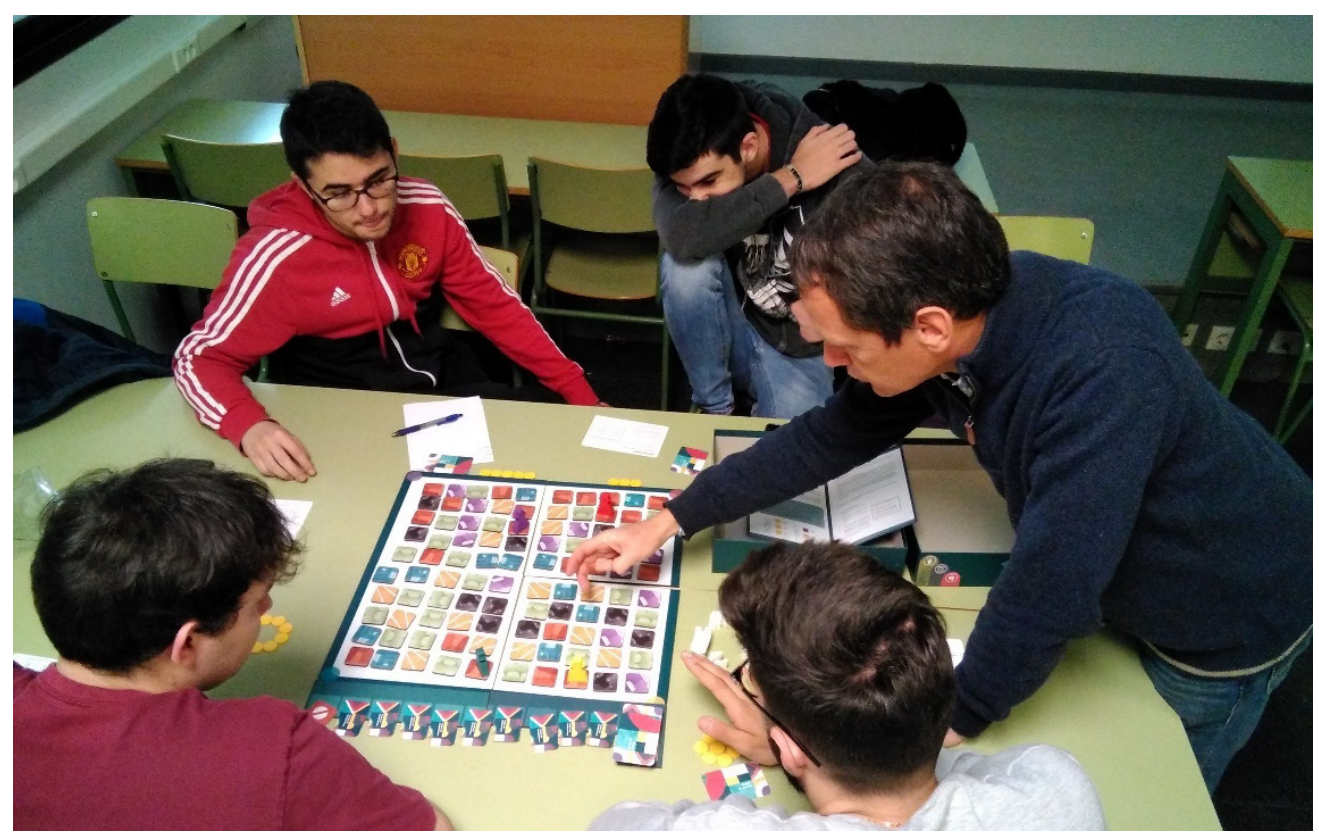

Figura 9: Mesa celebrada en la asignatura Transporte y Territorio, $2^{\circ}$ curso de Grado de Ingeniería Civil.

Entre los resultados más destacables de la encuesta realizada al alumnado tras las primeras sesiones de juego, se desprende que el alumnado reconoce haber trabajado e identificado diversas competencias relativas a la teoría explicada. Como se puede observar en la Figura 10, un $44,9 \%$ y un $38,8 \%$ estiman que se han trabajado mucho o bastante (lo que supone un 83,7\%), las competencias sobre ordenación del territorio siguientes:

- $\quad$ El territorio es un espacio de convivencia entre distintos actores sociales

- La toma de decisión en la Ordenación del Territorio es multicriterio

- Las características iniciales de un territorio suponen una serie de ventajas y desventajas comparativas

- $\quad$ El territorio es un espacio competitivo donde cada uno actúa guiado por sus propios intereses

- La localización de los equipamientos condiciona la localización de los usos del suelo

- Los usos del suelo condicionan la localización de los equipamientos

- El territorio es un espacio de conflictos entre intereses contrapuestos 


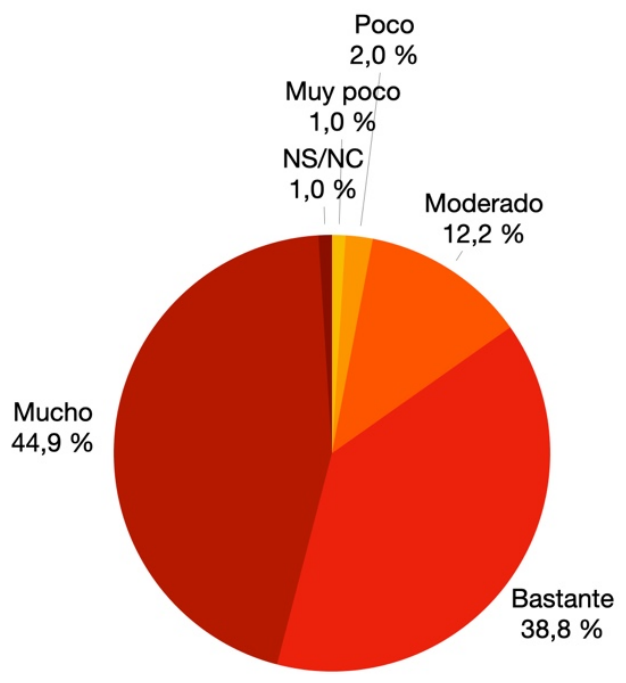

Figura 10: Valoración de los conceptos de Ordenación del Territorio trabajados en el juego

De la misma manera, el alumnado entiende criterios de ordenación del territorio básicos como la importancia de mantener un tejido urbano compacto, la ventaja que proporciona la proximidad de la huerta al tejido urbano, la importancia de construir redes de espacios naturales, de localizar el tejido urbano fuera de las zonas inundables o de la protección de los montes frente a incendios también son identificados, trabajados y conceptualizados en un $78,5 \%$ (un $37,1 \%$ mucho y un $41,4 \%$ bastante).

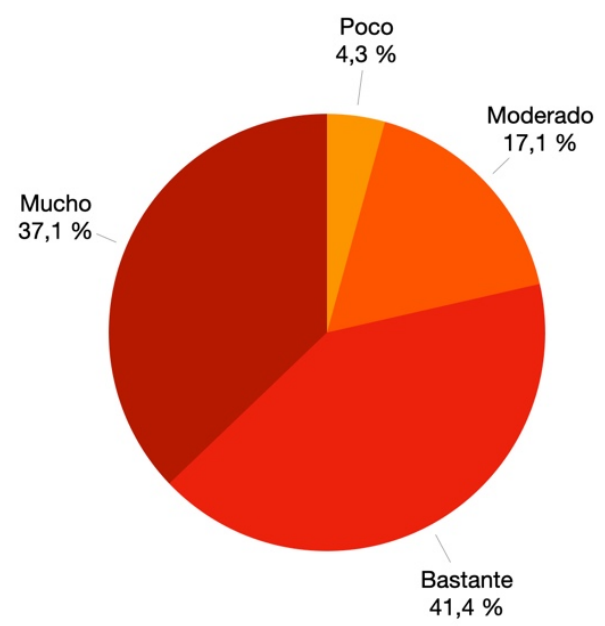

Figura 11: Valoración de los criterios de ordenación del territorio trabajados en el juego

Finalmente, como se desprende de la Figura 12, un 75\% (33,9\% mucho y un 41,1\% bastante) de los encuestados visualizan también en el juego conceptos asociados a la prestación de servicios de los equipamientos públicos como la importancia de disponer de bienes y servicios, en cantidad y calidad suficiente para satisfacer las demandas de la población, las diferencias existentes entre municipios en la accesibilidad a los servicios, la proximidad de la población a los servicios como fortaleza para un 
municipio o incluso las ideas de población atendida y desatendida por los equipamientos. En este caso, no es de extrañar unos porcentajes algo inferiores ya que reflejan conceptos cada vez más complejos.

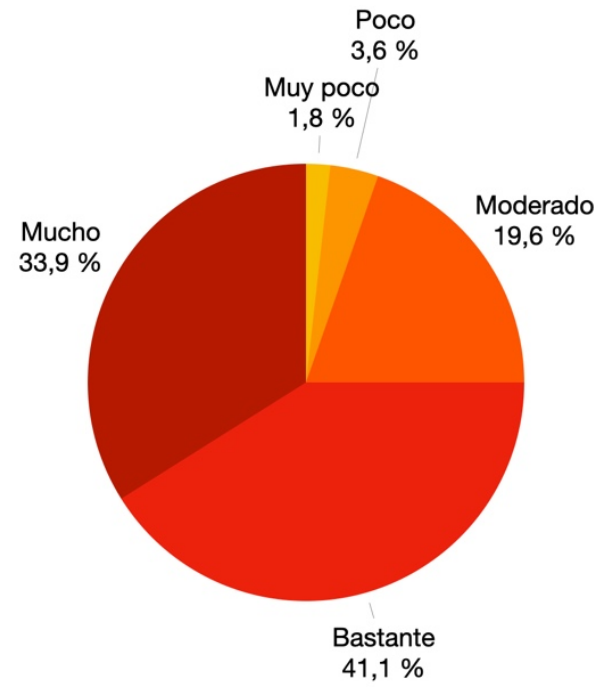

Figura 12: Valoración de los conceptos sobre equipamientos trabajados en el juego

En otro orden de cosas y corroborando las encuestas que se habían hecho ex ante del proyecto entre el alumnado de la ETSICCP, el alumnado reconoce que la utilización de recursos didácticos diferentes a las clases magistrales constituye un elemento motivador para el aprendizaje. Esto se ha podido constatar también con el proyecto transversal desarrollado en la Facultad de BBAA en el GDTTCC. En ambas actividades, el alumnado muestra, con su actitud durante la actividad y con sus comentarios a posteriori, un papel muy activo en la actividad que a su vez redunda en una motivación extra por aprender y trabajar. Algunos de estos comentarios fueron recogidos en entrevistas realizadas a los alumnos y alumnas de diseño, en concreto se realizaron 8 entrevistas a 4 alumnas y 4 alumnos de primero. En ellas los alumnos del GDTTCC detectaron algunos problemas de jugabilidad, ayudando a mejorar el producto final.

Por último, el propio diseño del juego, así como, el hecho de que el alumnado lo perciba como una posibilidad satisfactoria de sentirse protagonistas con la materia de estudio, ha cambiado por completo la percepción del aprendizaje. Los resultados ponen de manifiesto que después de una sorpresa inicial, el alumnado reconoce lo atractivo y útil que puede ser este tipo de recurso en el aula universitaria. Del mismo modo, se ha evidenciado que este recurso contribuye a generar un ambiente en el aula y una relación distinta con el profesor, más próxima, que propicia una mejora de la experiencia del aprendizaje. Con ello, el objetivo de "seducir" y atraer a los estudiantes hacia la disciplina del Urbanismo y la Ordenación del Territorio parece haberse conseguido.

\section{Conclusiones}

Desde hace varios años se ha producido una disminución de matrícula e interés por las carreras de ingeniería relacionadas con la obra civil, la construcción, el urbanismo y el territorio, lo que ha provocado que el perfil del alumnado que ingresa en estas carreras acuda con poca motivación. Por otro lado, las asignaturas del área de Ordenación del Territorio y Urbanismo involucrada en esta propuesta se caracterizan por tener un enfoque interdisciplinario y global, lo que supone la incorporación del pensamiento complejo, transversal y a menudo abstracto, en la enseñanza. Todo ello supone para el alumnado cierta dificultad en el aprendizaje y escasa motivación. El recurso lúdico La Comarca, que nace

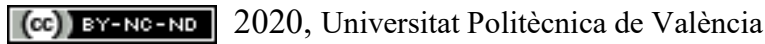

Congreso In-Red (2020) 
de un Proyecto de Innovación y Mejora Educativa financiado por el Vicerrectorado de Estudios, Calidad y Acreditación de la Universitat Politècnica de València, y por la Escuela Técnica Superior de Ingeniería de Caminos, Canales y Puertos, se plantea precisamente para paliar estos problemas.

La Comarca es un juego de estrategia de cooperación-oposición, diseñado para que el alumnado interactúe y trabaje con el objetivo de conseguir un territorio bien organizado. En opinión de los docentes participantes en el PIME, el marco definido en las reglas del juego reproduce los criterios básicos utilizados en el Urbanismo y la Ordenación del Territorio, en forma de decisión multicriterio, y los grupos compiten para cumplir, antes que las demás, sus objetivos, pero para ganar la partida es necesario que la "sociedad" construida alcance ciertos niveles de calidad y bienestar. Guiado por distintos criterios propuestos, cada uno de los participantes definirá y desarrollará su propia estrategia, en una partida en la que para conseguir sus objetivos se tendrá que buscar la colaboración de los demás participantes y, en otros momentos, se habrán de enfrentar entre ellos.

Los primeros resultados de los testeos iniciales de este proyecto muestran que el juego La Comarca puede ser un recurso didáctico útil, permitiendo trabajar y asimilar conceptos complejos con éxito (entre un $83,7 \%$ y 75\%), permitiendo presentar de manera sencilla muchos de los conceptos básicos de la disciplina, a veces difíciles de visualizar por el alumnado, pudiendo ser trabajados y asimilados de forma más natural. Se constata que este tipo de estrategias pedagógicas se pueden llevar a las aulas universitarias con éxito, en la que la acogida por parte del alumnado ha sido excelente. En definitiva, constituye una herramienta diferente y complementaria para construir una relación distinta profesoradoalumnado, así como motivar, acercar y enseñar conceptos básicos del Urbanismo y la Ordenación del Territorio en cualquiera titulación en la Universidad o incluso, fuera de ella.

\section{Referencias}

ALCAIDE, M․A., DE LA POZA, E. (2019). "El uso de los dispositivos electrónicos móviles como herramienta docente de una asignatura de Grado" en Congreso de Innovación Educativa y Docencia en Red de la Universitat Politècnica de València (Congreso In-Red). Valencia: Universitat Politècnica de València. 110-120.

CANO-TERrizA, D., ARENAS, A., BORGE, C., CARBOnERO, A., PANiAGUA, J., Risalde, M. A., ... \& GARCÍA-BOCANEGRA, I. (2019). "Gamificación como apoyo a la docencia en el Grado en Veterinaria". VetDoc. Revista de Docencia Veterinaria, 3, 111-112.

CASTAÑEDA-VÁZQUEZ, C., ESPEJO-GARCÉS, T., ZURITA-ORTEGA, F., \& FERNÁNDEZ-REVELLES, A. (2019). "La formación de los futuros docentes a través de la gamificación, tic y evaluación continua". SPORT TKRevista EuroAmericana De Ciencias Del Deporte, 8(2), 55-63.

CHACÓN, P. (2008). “El Juego Didáctico como estrategia de enseñanza y aprendizaje. ¿Cómo crearlo en el aula?” Nueva Aula Abierta, 16. Disponible en: http://www.grupodidactico2001.com/PaulaChacon.pdf

DELORS, J. (1996). "La educación encierra un tesoro". Informe a la UNESCO de la Comisión Internacional para la Educación del siglo XXI [en línea]. Ediciones UNESCO, Santillana

GAETE-QUEZADA, R.A. (2011). "El juego de roles como estrategia de evaluación de aprendizajes universitarios". Educación y Educadores, 14(2), 289-307

GONZÁLEZ GONZÁLEZ, C.S. (2014). "Estrategias para trabajar la creatividad en la Educación Superior: pensamiento de diseño, aprendizaje basado en juegos y en proyectos". RED - Revista de Educación a Distancia, nº40, 1-15, http://revistas.um.es/red/article/view/234291/180001

JUUL, J. (2003). "The Game, the Player, the World: Looking for a Heart of Gameness" en Proceedings of Level Up: Digital Games Research Conference, 30-45. 
PAISLEY, V. (2013). “Gamification of Tertiary Courses: An Exploratory Study of Learning and Engagement” en H. Carter, M. Gosper and J. Hedberg (Eds.), Electric Dreams. Proceedings ascilite 2013 Sydney. (pp.671-675).

PÉREZ VÁZQUEZ, E.; GILABERT CERDÁ, A.; LLEDÓ CARRERES, A. (2019) "Gamificación en la educación universitaria: El uso del escape room como estrategia de aprendizaje". Roig-Vila, Rosabel (ed.). Investigación e innovación en la Enseñanza Superior. Nuevos contextos, nuevas ideas. Barcelona: Octaedro, 2019. pp. 660-668

PIÑEIRO, R. (2016). "El boom de los juegos de mesa de autor" en El País. https://verne.elpais.com/verne/2016/11/07/articulo/1478519008_824740.html [Consulta: 04 de abril de 2020]

RÍOS, A. DE LOS, MUÑOZ, Y., CASTRO, P., ARROYO, J.L. (2019). "Gamification, strategy shared between university, company and millennials". REDU. Revista de Docencia Universitaria, 17(2), 73-88. https://doi.org/10.4995/redu.2019.11479

RIPOLL, O. (2006). “El juego como herramienta educativa”. Revista Educación Social, Vol. 33, pp. 11-27

RODRÍGUEZ, F., \& SANTIAGO, R. (2015). "Gamificación”. Cómo motivar a tu alumnado y mejorar el clima en el aula. Barcelona: Editorial Océano.

ROIG-VILA, R. (ed.) (2019). Investigación e innovación en la Enseñanza Superior. Nuevos contextos, nuevas ideas. Barcelona: Octaedro, 2019. 1297 p.

SERNA, E., DOLORES MAURICIO, M., SAN MIGUEL, T., MEGÍAS, J. (2016). "Experiencia de gamificación en Docencia Universitaria: aprendizaje activo y entretenido" en Congreso de Innovación Educativa y Docencia en Red de la Universitat Politècnica de València (Congreso In-Red). Valencia: Universitat Politècnica de València., pp. 364373.

SOSA ESPINOSA, A.; PEREZ ALONSO, Y. (2014). "ROLE PLAYING: Una buena herramienta para aprender" en Martí Selva, ML.; Calafat Marzal, MC. (2014). I Jornadas de Investigación de la Facultad de ADE. Editorial Universitat Politècnica de València. http://hdl.handle.net/10251/43966 